Journal Club

Editor's Note: These short, critical reviews of recent papers in the Journal, written exclusively by graduate students or postdoctoral fellows, are intended to summarize the important findings of the paper and provide additional insight and commentary. For more information on the format and purpose of the Journal Club, please see http://www.jneurosci.org/misc/ifa_features.shtml.

\title{
Evolutionarily Conserved Mechanisms in Calcium Handling May Underlie Intrinsic Sensitivity to Dopaminergic Neuron Death
}

\author{
(1)Daniel G. Taub \\ Department of Physiology and Biophysics, Boston University School of Medicine, Boston, Massachusetts 02218 \\ Review of Nagarajan et al.
}

Mammalian dopaminergic neurons of the substantia nigra pars compacta $(\mathrm{SNc})$ within the CNS are complex neurons with expansive arborization. Projecting from the SNc to the striatum, the average total axonal length of these neurons in the rat has been estimated to be $\sim 467 \mathrm{~mm}$, with each axon supporting upwards of 370,000 synapses (Matsuda et al., 2009). This massive network contributes to our ability to execute movement and degeneration of these neurons has catastrophic effects, as seen in Parkinson's disease (PD).

Many hypotheses have been put forth to explain the death of dopaminergic neurons in $\mathrm{PD}$, including pathological aggregation of $\alpha$-synuclein (a component of Lewy bodies), calcium toxicity, and lysosomal and mitochondrial dysfunction (Sulzer, 2007). Furthermore, dopamine/ $\alpha$ synuclein interactions have been suggested as a potential pathogenic mechanism in which dopamine can modify $\alpha$-synuclein to increase its reactivity and aggregative properties. In turn, the reactive aggregation may disrupt the membrane of dopamine-containing vesicles, leading to higher cytoplasmic dopamine levels and further $\alpha$-synuclein modification, or potentially reach cytotoxic levels of dopa-

Received June 2, 2014; revised July 9, 2014; accepted July 14, 2014.

Correspondence should be addressed to Daniel G. Taub, Department of

Physiology and Biophysics, Boston University School of Medicine, 72 East Concord Street, Boston, MA 02218. E-mail: dgtaub@bu.edu.

DOI:10.1523/JNEUROSCI.2240-14.2014

Copyright $\odot 2014$ the authors $\quad 0270-6474 / 14 / 3410795-03 \$ 15.00 / 0$ mine, although this hypothesis has just begun to be rigorously tested (Sulzer, 2007; Mosharov et al., 2009). Recent observations in mouse models of PD suggest that interplay between these pathologies might be responsible for dopamingeric cell death of the SNc (Sulzer, 2007). Interestingly, although dopaminergic neurons in both the SNc and the ventral tegmental area (VTA) exhibit Lewy bodies, the neurons of the SNc tend to undergo degradation while those of the VTA are relatively spared (Dauer and Przedborski, 2003).

Why are VTA neurons spared the degenerative fate of SNc neurons? Calcium toxicity has long been proposed as a mechanism in neuronal cell death, including necrosis and apoptosis. Maintenance of low intracellular calcium levels is essential for cellular function and survival. Interestingly, the calcium binding protein, calbindin, is differentially expressed between VTA and SNc neurons, and single nucleotide polymorphisms in the human calbindin gene, CALB1, have been associated with sporadic PD in a Japanese cohort (Mizuta et al., 2008). Calbindin contains five EF-hand protein motifs that are thought to buffer cytoplasmic calcium levels, thus serving a neuroprotective function. High expression of calbindin is observed in the VTA while the ventral $\mathrm{SNc}$ is calbindin-negative (German et al., 1992). This suggests that neurons of the VTA are better equipped to handle fluctuations in intracellular cal- cium. Differences in other calciumbinding proteins, such as calretinin, also appears to convey a neuroprotective function in some dopaminergic neurons (Mouatt-Prigent et al., 1994).

Caenorhabditis elegans has emerged as a powerful tool to address the molecular mechanisms underlying PD and has provided essential information regarding dopaminergic neuronal degeneration (Harrington et al., 2010). In a recent edition of The Journal of Neuroscience, Nagarajan et al. (2014) performed a mutagenesis screen in C. elegans to identify genes that are involved in the progressive loss of dopaminergic neurons. Nagarajan et al. (2014) identified a mutation in an essential pore forming subunit of a transient receptor potential (TRP) channel that allowed excess calcium to enter dopaminergic neurons, leading to necrotic-like cell death. Nagarajan et al.'s (2014) findings may therefore shed light on the vulnerability of dopaminergic neurons.

Nagarajan et al. (2014) found that $C$. elegans mutants that had gain-of-function mutations in the trp-4 gene (which they termed trp-4(d)) displayed normal development of dopaminergic neurons, but dopaminergic cell death during adulthood. Although there are no direct mammalian homologs of TRP4, many TRP channels are present in mammalian dopaminergic neurons of the SNc, suggesting common functionality may exist (Riccio et al., 2002). The role of TRP channels in 
PD is still unclear, but several observations suggest TRPC1 may be involved in dopaminergic endoplasmic reticulum (ER) stress and death (Selvaraj et al., 2010).

As most all TRPs are permeable to calcium ions, Nagarajan et al. (2014) hypothesized that dysregulation of calcium signaling and calcium handling caused the neuronal degeneration. They noted that the phenotype of $\operatorname{trp}-4(d)$ mutants was strikingly similar to a previously described gain-of-function mutation in a degenerin/epithelial sodium channel subunit, mec-4 (Royal et al., 2005). In nematodes with an A713V mutation in mec-4 (termed mec-4(d)), specific mechanosensory neurons develop normally, but undergo degeneration via calcium-mediated necrosis by adulthood (Royal et al., 2005). Growth of $m e c-4(d)$ mutants in the presence of a calcium chelator and genetic alterations of calcium handling pathways restored neuronal survival. Therefore, Nagarajan et al. (2014) grew trp-4(d) mutants in the presence of a calcium chelator and observed a mild rescue of neuronal degeneration. Furthermore, double mutants of $\operatorname{trp}-4(d)$ and calreticulin (crt-1), a calcium-binding endoplasmic reticulum chaperone, significantly increased neuronal survival. $\operatorname{trp}-4(d)$;itr-1 and $\operatorname{trp}-4(d)$; unc-68 double mutants, which abolish inositol triphosphate (IP3) and ryanodine receptors of the ER, respectively, also displayed a mild increase in survival. Although the authors did not directly measure intracellular calcium levels, these findings indicate that intracellular calcium and, in particular, the mobilization of intracellular stores are necessary for the observed degeneration.

The work of Nagarajan et al. (2014) clearly illustrates that $\operatorname{trp}-4(d)$ dopaminergic neurons undergo a form of necrotic degeneration similar to that of mec-4(d) mutants. As a next step, it would be interesting to confirm the role of the $\operatorname{trp}-4(d)$ in calcium permeability by expressing in Xenopus embryos and performing electrophysiological measurements, as done previously for the mec-4(d) mutant channel (Royal et al., 2005). Alternatively, calcium imaging within C. elegans using a genetically encoded calcium indicator could be used to directly measure calcium mishandling as the causal factor. As mitochondria are also an important store of intracellular calcium, it would be interesting to address their role in this calcium-mediated toxicity as well.

As mentioned previously, one feature of PD is the specific degeneration of dopa- minergic neurons in the SNc while neurons of the VTA, and other populations, are relatively spared. Likewise, Nagarajan et al. (2014) show that not all dopaminergic neurons are equally sensitive to degeneration. While the head neurons [cephalic (CEP) and anterior deirid (ADE) neurons] of $\operatorname{trp}-4(d)$ mutants often underwent necrosis, the tail neurons of males and posterior deirid (PDE) neurons tended not to degenerate. This difference is somewhat mitigated by overexpression of the $\operatorname{trp}-4(d)$ mutant gene, which can induce cell death in nearly all dopaminergic cells. Nonetheless, PDE neurons remain more resilient even in transgenic $\operatorname{trp}$-4(d)-overexpressing nematodes, with less frequent degeneration occurring than in the CEP neurons. Similarly, CEP neurons were much more susceptible than PDE neurons to 6-OHDA, a synthetic reagent that selectively kills dopaminergic neurons and is often used to induce PDlike symptoms in animal models (Nass et al., 2002). What cellular differences cause this difference in sensitivity? Nagarajan et al. (2014) suggest that calcium regulation may lie at the heart of this effect. Further elucidation of calcium-mediated degeneration pathways should begin to address differential vulnerability of specific neurons to calcium. In addition, examining the expression patterns of different calcium-binding proteins in different neurons in $C$. elegans will be an essential follow up experiment.

The finding that dopaminergic neurons are sensitive to fluctuations in intracellular calcium is not new. In fact, calcium transients have been recognized as a potential cause of mitochondrial stress in dopaminergic neurons of the mammalian SNc (Chan et al., 2007). The dopaminergic neurons of the SNc display autonomous pacemaking behavior in the absence of presynaptic influence with a firing frequency between 1 and $4 \mathrm{~Hz}$ (Grace and Bunney, 1984). In conjunction with neuronal firing, intracellular levels of calcium spike by activation of L-type voltage-gated calcium channels (Guzman et al., 2010). The function of the pacemaking activity is likely to sustain dopamine concentrations in the striatum (Romo and Schultz, 1990). The oscillations in intracellular calcium challenge dopaminergic neurons, because calcium levels must be tightly regulated. This challenge can be observed in mice with a mitochondrial-specific green fluorescent protein that fluoresces during oxidative stress. Dopaminergic neurons of the $\mathrm{SNc}$ display much higher basal levels of oxida- tive stress than neurons of the VTA. Furthermore, treatment of SNc neurons with isradipine, a calcium-channel blocker, reduces the levels of oxidative stress indicating that increases in intracellular calcium are responsible (Chan et al., 2007). On top of this constant pacemaking activity, dopaminergic neurons are stimulated by glutamatergic inputs, which further drives calcium levels within the cell through the NMDA receptor (Deister et al., 2009). It is easy to envision that even a slight dysregulation in calcium handling can be potentially toxic to these cells and differential sensitivity between dopaminergic neurons may influence the decision to undergo cell death.

By demonstrating that dopaminergic neurons in C. elegans display differential sensitivity to $\operatorname{trp}-4(d)$-mediated cell death, Nagarajan et al. (2014) provide a potential window into calcium-mediated degeneration of dopaminergic neurons. It is important to note that despite the relative simplicity of C. elegans dopaminergic neurons, including lack of action potentials, simple morphologies, and synaptic connections, their function remains somewhat conserved, as both mammalian and C. elegans dopaminergic neurons play an integral role in coordinating locomotion (Chase and Koelle, 2007). The findings by Nagarajan et al. (2014) suggest that evolutionarily conserved mechanisms may underlie the intrinsic sensitivity of dopaminergic neurons and this sensitivity is greatly modulated by neuronal-specific calcium handling abilities. Future work on differential calcium sensitivity of neuronal populations using this $\operatorname{trp}-4(d)$ mutant will have the advantage of the high-throughput, genetically accessible C. elegans model system to further our understanding of the process of dopaminergic neuronal degeneration. This will undoubtedly provide exciting insights into the vulnerability of certain neurons to cell death.

\section{References}

Chan CS, Guzman JN, Ilijic E, Mercer JN, Rick C, Tkatch T, Meredith GE, Surmeier DJ (2007) 'Rejuvenation' protects neurons in mouse models of Parkinson's disease. Nature 447: 1081-1086. CrossRef Medline

Chase DL, Koelle MR (2007) Biogenic amine neurotransmitters in C. elegans. WormBook: $1-15$.

Dauer W, Przedborski S (2003) Parkinson's disease: mechanisms and models. Neuron 39: 889-909. CrossRef Medline

Deister CA, Teagarden MA, Wilson CJ, Paladini CA (2009) An intrinsic neuronal oscillator underlies dopaminergic neuron bursting. J Neurosci 29:15888-15897. CrossRef Medline 
German DC, Manaye KF, Sonsalla PK, Brooks BA (1992) Midbrain dopaminergic cell loss in Parkinson's disease and MPTP-induced parkinsonism: sparing of calbindin-D28kcontaining cells. Ann N Y Acad Sci 648:42-62. CrossRef Medline

Grace AA, Bunney BS (1984) The control of firing pattern in nigral dopamine neurons: single spike firing. J Neurosci 4:2866-2876. Medline

Guzman JN, Sanchez-Padilla J, Wokosin D, Kondapalli J, Ilijic E, Schumacker PT, Surmeier DJ (2010) Oxidant stress evoked by pacemaking in dopaminergic neurons is attenuated by DJ-1. Nature 468:696-700. CrossRef Medline

Harrington AJ, Hamamichi S, Caldwell GA, Caldwell KA (2010) C. elegans as a model organism to investigate molecular pathways involved with Parkinson's disease. Dev Dyn 239: 1282-1295. CrossRef Medline

Matsuda W, Furuta T, Nakamura KC, Hioki H, Fujiyama F, Arai R, Kaneko T (2009) Single nigrostriatal dopaminergic neurons form widely spread and highly dense axonal arborizations in the neostriatum. J Neurosci 29: 444-453. CrossRef Medline

Mizuta I, Tsunoda T, Satake W, Nakabayashi Y,
Watanabe M, Takeda A, Hasegawa K, Nakashima K, Yamamoto M, Hattori N, Murata M, Toda T (2008) Calbindin 1, fibroblast growth factor 20, and alpha-synuclein in sporadic Parkinson's disease. Hum Genet 124: 89-94. CrossRef Medline

Mosharov EV, Larsen KE, Kanter E, Phillips KA, Wilson K, Schmitz Y, Krantz DE, Kobayashi K, Edwards RH, Sulzer D (2009) Interplay between cytosolic dopamine, calcium, and alpha-synuclein causes selective death of substantia nigra neurons. Neuron 62:218-229. CrossRef Medline

Mouatt-Prigent A, Agid Y, Hirsch EC (1994) Does the calcium binding protein calretinin protect dopaminergic neurons against degeneration in Parkinson's disease? Brain Res 668: 62-70. CrossRef Medline

Nagarajan A, Ning Y, Reisner K, Buraei Z, Larsen JP, Hobert O, Doitsidou M (2014) Progressive degeneration of dopaminergic neurons through TRP channel-induced cell death. J Neurosci 34:5738-5746. CrossRef Medline

Nass R, Hall DH, Miller DM 3rd, Blakely RD (2002) Neurotoxin-induced degeneration of dopamine neurons in Caenorhabditis elegans.
Proc Natl Acad Sci U S A 99:3264-3269. CrossRef Medline

Riccio A, Medhurst AD, Mattei C, Kelsell RE, Calver AR, Randall AD, Benham CD, Pangalos MN (2002) mRNA distribution analysis of human TRPC family in CNS and peripheral tissues. Brain Res Mol Brain Res 109:95-104. CrossRef Medline

Romo R, Schultz W (1990) Dopamine neurons of the monkey midbrain: contingencies of responses to active touch during self-initiated arm movements. J Neurophysiol 63:592-606. Medline

Royal DC, Bianchi L, Royal MA, Lizzio M Jr, Mukherjee G, Nunez YO, Driscoll M (2005) Temperature-sensitive mutant of the Caenorhabditis elegans neurotoxic MEC-4(d) DEG/ $\mathrm{ENaC}$ channel identifies a site required for trafficking or surface maintenance. J Biol Chem 280:41976-41986. CrossRef Medline

Selvaraj S, Sun Y, Singh BB (2010) TRPC channels and their implication in neurological diseases. CNS Neurol Disord Drug Targets 9:94-104. CrossRef Medline

Sulzer D (2007) Multiple hit hypotheses for dopamine neuron loss in Parkinson's disease. Trends Neurosci 30:244-250. CrossRef Medline 